

\title{
Threshold MnAs thickness for the formation of ordered $\alpha / \beta$ stripes in MnAs/GaAs(001)
}

Maurizio Sacchi, Nicolas Casaretto, Leticia Coelho, Mahmoud Eddrief, Jialin Ma, Carlo Spezzani, Franck Vidal, Hailong Wang, Jianhua Zhao, Yunlin Zheng

\section{To cite this version:}

Maurizio Sacchi, Nicolas Casaretto, Leticia Coelho, Mahmoud Eddrief, Jialin Ma, et al.. Threshold MnAs thickness for the formation of ordered $\alpha / \beta$ stripes in MnAs/GaAs(001). Journal of Physics D: Applied Physics, 2020, 53 (26), pp.265005. 10.1088/1361-6463/ab82da . hal-03028454

\section{HAL Id: hal-03028454 \\ https://cnrs.hal.science/hal-03028454}

Submitted on 27 Nov 2020

HAL is a multi-disciplinary open access archive for the deposit and dissemination of scientific research documents, whether they are published or not. The documents may come from teaching and research institutions in France or abroad, or from public or private research centers.
L'archive ouverte pluridisciplinaire HAL, est destinée au dépôt et à la diffusion de documents scientifiques de niveau recherche, publiés ou non, émanant des établissements d'enseignement et de recherche français ou étrangers, des laboratoires publics ou privés. 


\title{
Threshold MnAs thickness for the formation of ordered $\alpha / \beta$ stripes in $\operatorname{MnAs} / \mathbf{G a A s}(001)$
}

\author{
Maurizio Sacchi ${ }^{1,2, *}$, Nicolas Casaretto ${ }^{1}$, Leticia Coelho ${ }^{1, \dagger}$, Mahmoud Eddrief ${ }^{1}$, Jialin $\mathrm{Ma}^{3}$, \\ Carlo Spezzani $^{4}$, Franck Vidal ${ }^{1}$, Hailong Wang ${ }^{3}$, Jianhua Zhao ${ }^{3}$, Yunlin Zheng ${ }^{1}$ \\ ${ }^{1}$ Sorbonne Université, CNRS, Institut des NanoSciences de Paris, INSP, \\ F-75005 Paris, France \\ ${ }^{2}$ Synchrotron SOLEIL, L'Orme des Merisiers, Saint-Aubin, \\ B.P. 48, 91192 Gif-sur-Yvette, France \\ ${ }^{3}$ State Key Laboratory of Superlattices and Microstructures, Institute of Semiconductors, \\ Chinese Academy of Sciences, P. O. Box 912, Beijing 100083, P. R. China \\ ${ }^{4}$ ELETTRA, Sincrotrone Trieste, Area Science Park, \\ S.S.14, Km 163.5, 34012 Trieste, Italy
}

\begin{abstract}
Manganese arsenide layers epitaxially grown on $\mathrm{GaAs}(001)$ are known to feature a temperature dependent self-assembled microstructure of ordered stripes, alternating the ferromagnetic $\alpha$ and paramagnetic $\beta$ phases. The surface dipolar fields generated by the $\alpha / \beta$ stripes have been used for achieving temperature controlled magnetization switching of a ferromagnetic overlayer. For this kind of application, it is advantageous to minimize the MnAs layer thickness. In this work we investigate, using $\mathrm{x}$-ray scattering techniques, the presence of the ordered microstructure as a function of the MnAs layer thickness and we identify a minimum value of $\sim 40 \mathrm{~nm}$ for the formation of ordered $\alpha / \beta$ stripes in $\mathrm{MnAs} / \mathrm{GaAs}(001)$. These results have an impact for envisaging magnetization-switching applications that rely on the control of the temperature- or laser-driven surface dipolar fields in MnAs-based devices.
\end{abstract}

*e-mail: maurizio.sacchi@insp.jussieu.fr

${ }^{\dagger}$ Prmanent address: Instituto de Física, Universidade de Brasília UnB, Brasília - DF, CEP 70910-900, Brazil 


\section{INTRODUCTION}

Developing thermally induced magnetization switching (TIMS), where the temperature control of intrinsic material properties drives a deterministic reversal of the local magnetization, has potential for applications. For instance, the combination of materials with different temperature dependent magnetic properties has been used to switch the magnetization with a single laser pulse in rare-earth-transition-metal ferrimagnetic compounds, in a process that combines thermal effects with photon helicity dependent effects [1-4]. Controlling the local temperature by a laser pulse is also at the basis of heat assisted magnetic recording processes that were proposed to significantly lower the external field values necessary for switching a magnetic bit [5]. A different approach to TIMS makes use of substrates whose intrinsic magnetic properties feature regular spatial modulations generating local surface dipolar fields that can be controlled by tuning the temperature [6]. The proof-of-principle of this approach made use of a thin ferromagnetic (FM) Fe layer deposited on a MnAs/GaAs(001) template. MnAs/GaAs(001) is a metal/semiconductor system where the interplay between epitaxial constraints and temperature-driven phase transitions gives rise to a peculiar morphological and magnetic behavior at ambient temperatures $[7,8]$. At $\mathrm{T} \sim 40^{\circ} \mathrm{C}$, bulk MnAs features a first order transition between ferromagnetic $\alpha$ and non-magnetic $\beta$ phases [9-11]. In MnAs/GaAs(001) epitaxial films the two phases coexist over the temperature range $\sim 10-40{ }^{\circ} \mathrm{C}$ in the form of regular stripes $[7,12]$ the period of which is determined by the MnAs thickness $[7,8,13]$ and by the presence of overlayers [14]. It was shown that it is possible to control the Fe magnetization in $\mathrm{Fe} / \mathrm{MnAs} / \mathrm{GaAs}(001)$ by fine-tuning the temperature at ambient [15]. Element selective $\mathrm{x}$-ray resonant magnetic scattering (XRMS) experiments demonstrated that various magnetic configurations of the Fe/MnAs system can be stabilized predictably by acting on the thermal cycle parameters and on the presence of a bias field [16]. What one actually does by changing the temperature is to control the surface dipolar fields associated with the template magnetic microstructure, and, ultimately, vary the effective magnetic field acting on the overlayer. Time-resolved experiments showed the potential of this approach for fast magnetization switching, where the microstructure changes are triggered by a single femtosecond optical laser pulse $[17,18]$.

The general motivation for this research is to develop a MnAs based two-layer systems for TIMS, where the overlayer magnetization can be switched by temperature control of the dipolar fields generated by the MnAs template. Up to now, TIMS was achieved by employing MnAs 
layers 100 to $200 \mathrm{~nm}$ thick, but our present understanding of the TIMS process in Fe/MnAs suggests that the advantage of using thinner MnAs layers is at least twofold. Firstly, the stripe period $\mathrm{p}$ is proportional to the film thickness $\mathrm{t}_{\mathrm{MnAs}}$, with $\mathrm{p} \approx 5 \times \mathrm{t}_{\mathrm{MnAs}}$ for a bare MnAs film [7, 8], therefore reducing $\mathrm{t}_{\mathrm{MnAs}}$ leads to narrower stripes that can generate stronger surface dipolar fields. A shorter period should also favor a better spatial definition of the TIMS process, since the size of the smallest bit of the overlayer that one can envisage switching independently is proportional to the period. Secondly, it was shown that a fluence threshold exists for laser induced switching, which corresponds to the complete transition of the MnAs film into the high temperature $\beta$ phase $[17,18]$. Therefore a thinner MnAs template should make it possible to switch the overlayer magnetization using lower laser fluence, i.e. a reduced power. Finally, since the characteristic time for magnetization switching is determined by the return towards thermal equilibrium [19], using a thinner template and a lower fluence will lead to a faster switching process.

In summary, reducing the MnAs layer thickness should allow for a faster laser-driven magnetization switching of smaller areas employing a lower power.

The controlled generation and disappearance of surface dipolar fields remains a pre-requisite for TIMS, which for MnAs implies the formation of regular $\alpha / \beta$ stripes. The occurrence of spatially modulated phase coexistence in thin films has been addressed in theoretical studies. In particular, the work of Sridar et al. [20], which was used for modelling the MnAs/GaAs(001) system [13], suggested that, for certain sets of parameters, the formation of regular stripes becomes unfavorable below a critical thickness of the film. Experimental studies exist on thin MnAs layers on $\mathrm{GaAs}(001)$, demonstrating that the crystal structure is well defined and relaxed for films a few nm thick [21] and that the $\alpha / \beta$ phase coexistence as a function of temperature is observed already for very thin films [22]. Other studies [23-27] report, albeit with some contradictory results, images of the local magnetic order in thin $(<50 \mathrm{~nm}) \mathrm{MnAs}$ layers. Finally, the role of the layer thickness has been evoked also in connection with the electronic and magnetic properties of $\sim \mu$ m-sized laterally confined MnAs ribbons [27, 28]. In any case, none of these studies addressed specifically the formation of ordered $\alpha / \beta$ stripes versus thickness in the limit of very thin MnAs layers.

In this work we employ resonant soft x-ray scattering to study the ordered microstructure as well as the magnetic properties of thin MnAs films. We analyze MnAs/GaAs(001) layers with thickness in the 20-70 $\mathrm{nm}$ range. The main objective is to determine the minimum thickness capable of producing a regular microstructure of $\alpha / \beta$ stripes that can generate at precise and predictable locations the surface dipolar fields necessary for TIMS. Our work confirms the 
existence of a threshold thickness for stripe formation; we discuss its possible origin in the framework of previous results reported in the literature.

\section{EXPERIMENTAL DETAILS}

MnAs layers 20 to $70 \mathrm{~nm}$ thick were prepared by molecular beam epitaxy (MBE) on GaAs(001) at the State Key Laboratory of Superlattices and Microstructures of the Institute of Semiconductors (IoS) in Beijing, following a well-established protocol [29, 30]. The samples were capped by $\sim 4 \mathrm{~nm}$ of GaAs for protection against contamination. As a reference, we measured also two samples prepared at the Institut des NanoSciences de Paris (INSP) that were used in previous experiments, one $30 \mathrm{~nm}$ thick [27], the other $180 \mathrm{~nm}$ thick [19]. All samples were characterized by x-ray diffraction (XRD) measurements at INSP, using the $\mathrm{Cu}-\mathrm{K} \alpha_{1}$ radiation from a rotating anode diffractometer equipped with a $\mathrm{Ge}(220)$ 2-reflection channel-cut monochromator. XRD data confirmed the growth of type-A MnAs layers [7], with the epitaxial relationship between MnAs and GaAs sketched in figure 1(a).

Soft $\mathrm{x}$-ray resonant scattering measurements were performed at the Circular Polarization beamline of the ELETTRA synchrotron (Trieste), using the IRMA scattering chamber [31]. The sample holder was equipped for temperature and magnetic field control. A photodiode with a $0.2 \mathrm{~mm}$ vertical aperture slit was placed on the detector arm at $150 \mathrm{~mm}$ from the sample. An in vacuum 2D detector, based on microchannel plates and a resistive anode, was mounted on a fixed flange (central scattering angle $2 \theta \approx 32.5^{\circ}$ ) and allowed us to collect the diffuse intensity around the specular beam and the diffraction peaks originating from the regular stripe microstructure. Scattering measurements were performed in co-planar geometry [see figure 1(b)], tuning the soft $\mathrm{x}$-ray energy to the $\mathrm{Mn}-2 \mathrm{p}$ resonance $(\sim 640 \mathrm{eV})$. In addition to providing a stronger diffraction signal from the ordered stripes, working at the Mn core resonance and using circularly polarized radiation allowed us to probe also the magnetic response as a function of temperature under the same experimental conditions.

The formation of regular stripes alternating the $\alpha$ and $\beta$ phases was monitored by probing the scattered intensity around the specular beam either by using the $2 \mathrm{D}$ detector or by performing sample rocking scans using the slitted photodiode. In both cases, the regular stripes produce Bragg peaks the angular location of which depends on the stripe period $\mathrm{p}$, on the $\mathrm{x}$-ray wavelength $\lambda$, and on the incidence $(\omega)$ and scattering $(2 \theta)$ angles. 


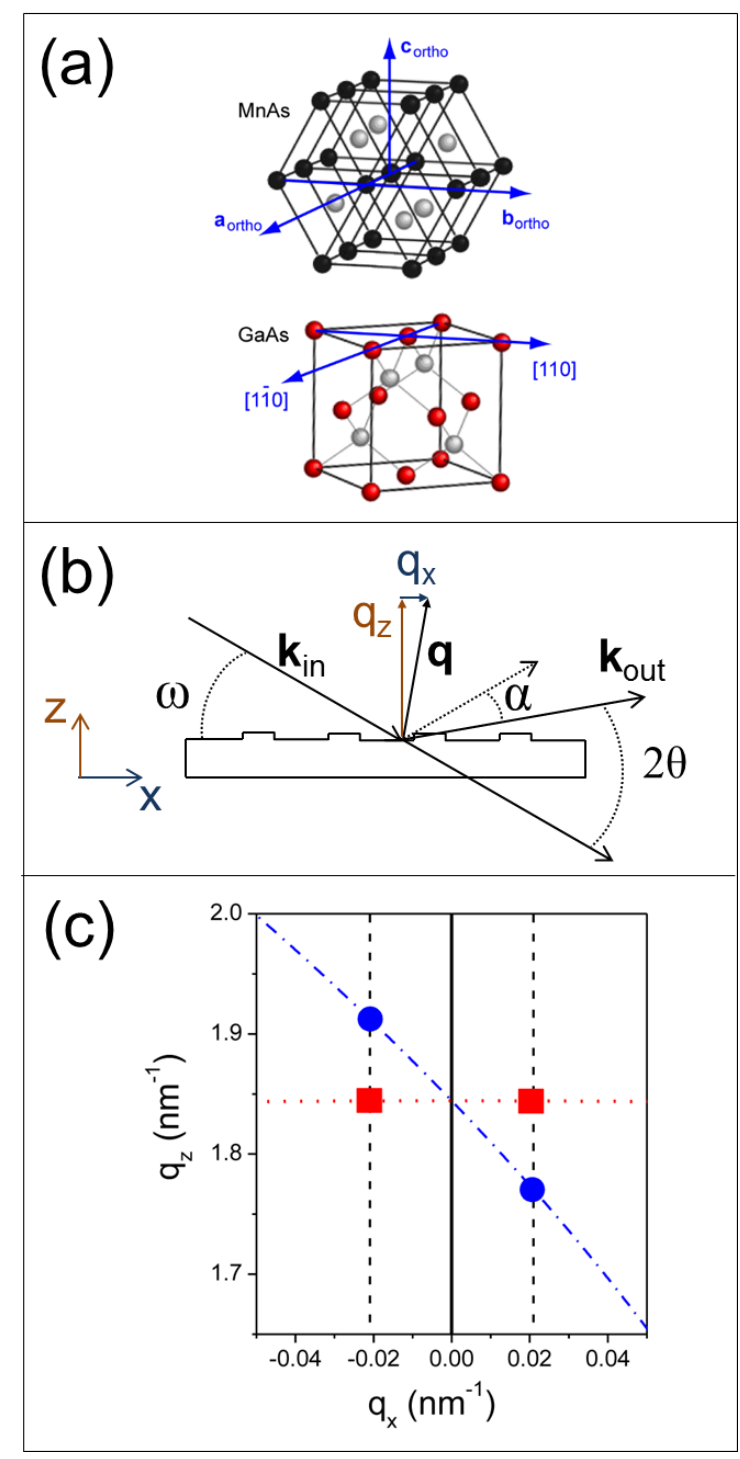

Figure 1. (a) Epitaxial relationship between MnAs (orthorhombic notation) and $\mathrm{GaAs}(001)$ in type-A growth. (b) Sketch of the scattering geometry, showing the incidence $(\omega)$, scattering $(2 \theta)$ and off-specular $(\alpha)$ angles. $\mathbf{k}_{\text {in }}$ and $\mathbf{k}_{\text {out }}$ represent the vector momentum of the incoming and outgoing photons, $\mathbf{q}=\mathbf{k}_{\text {out }}-\mathbf{k}_{\text {in }}$ is the exchanged momentum with components $q_{x}$ and $q_{z}$. (c) Schematic $\left(q_{x}, q_{z}\right)$ chart, assuming $\mathrm{p}=300 \mathrm{~nm}$ and $\lambda=1.94 \mathrm{~nm}$. The thick black line at $\mathrm{q}_{\mathrm{x}}=0$ corresponds to specular reflectivity $(\omega / 2 \theta$ scan with $\alpha=0)$. The red dotted line represents a rocking curve $(\omega$ scan at $2 \theta=$ $32.5^{\circ}$ ). The blue dash-dot line corresponds to a detector scan $\left(2 \theta\right.$ scan at $\left.\omega=16.25^{\circ}\right)$. The dashed vertical lines correspond to $\mathrm{q}_{\mathrm{x}}= \pm 2 \pi / \mathrm{p}= \pm 0.021$ $\mathrm{nm}^{-1}$. Large squares and circles locate the expected Bragg peaks on the rocking and detector scans, respectively.

Taking (x,y) as the sample surface and $(\mathrm{x}, \mathrm{z})$ as the incidence plane [see figure 1(b)], the exchanged momentum vector has components $\mathrm{q}_{\mathrm{x}}=(4 \pi / \lambda) \cdot \sin (\theta) \cdot \sin (\alpha), \quad \mathrm{q}_{\mathrm{y}}=0$ and $\mathrm{q}_{\mathrm{z}}=(4 \pi / \lambda) \cdot \sin (\theta) \cdot \cos (\alpha)$, where $\alpha=\omega-\theta$ is the sample off-specular angle. Figure $1(\mathrm{c})$ shows schematically in a $\left(\mathrm{q}_{\mathrm{x}}, \mathrm{q}_{\mathrm{z}}\right)$ chart the location of Bragg peaks at $\mathrm{q}_{\mathrm{x}}=2 \pi / \mathrm{p}$ for a stripe period $\mathrm{p}=300$ $\mathrm{nm}$, observed with a photon energy of $640 \mathrm{eV}(\lambda=1.94 \mathrm{~nm}, \mathrm{Mn}-2 \mathrm{p}$ resonance). On the same chart we have highlighted the lines corresponding to a detector scan and to a sample rocking scan. The former (blue dash-dot line) corresponds to a $2 \theta$ scan at fixed $\omega$ (in our case, to a $2 \mathrm{D}$-detector acquisition), the latter (red dotted line) to a $\omega$ scan at fixed $2 \theta$ (i.e., to a $\mathrm{q}_{\mathrm{x}}$ scan at almost constant $\left.\mathrm{q}_{\mathrm{z}}\right)$. In our measurements we could also cover whole areas of the $\left(\mathrm{q}_{\mathrm{x}}, \mathrm{q}_{\mathrm{z}}\right)$ chart by combing $\omega$ scans collected at different $2 \theta$ angles, using the slitted diode detector. 
Table 1 lists all the samples addressed specifically in this study and compares their nominal MnAs thickness values with those obtained by soft (synchrotron) and hard $(\mathrm{Cu}-\mathrm{K} \alpha)$ x-ray reflectivity. In the following, we will refer to each sample by its nominal thickness value.

\begin{tabular}{|c|c|c|c|c|}
\hline $\mathrm{t}_{\text {nominal }}(\mathrm{nm})$ & $\mathrm{t}_{\mathrm{SXR}}(\mathrm{nm})$ & $\mathrm{t}_{\mathrm{HXR}}(\mathrm{nm})$ & Laboratory & References \\
\hline 20 & 20 & 23 & IoS & \\
\hline 35 & 35 & 34 & IoS & \\
\hline 50 & 47 & 49 & IoS & \\
\hline 70 & 70 & 72 & IoS & [30] \\
\hline 30 & 29 & 29 & INSP & [27] \\
\hline 180 & 180 & 179 & INSP & [19] \\
\hline
\end{tabular}

\section{RESULTS}

Figure 2 shows for all samples the temperature dependence of the Mn magnetization in a heating cycle, measured by Mn-2p resonant magnetic scattering. The magnetic signal is defined as the difference between the scattered intensity recorded for opposite magnetization/helicity orientations divided by their sum [16].

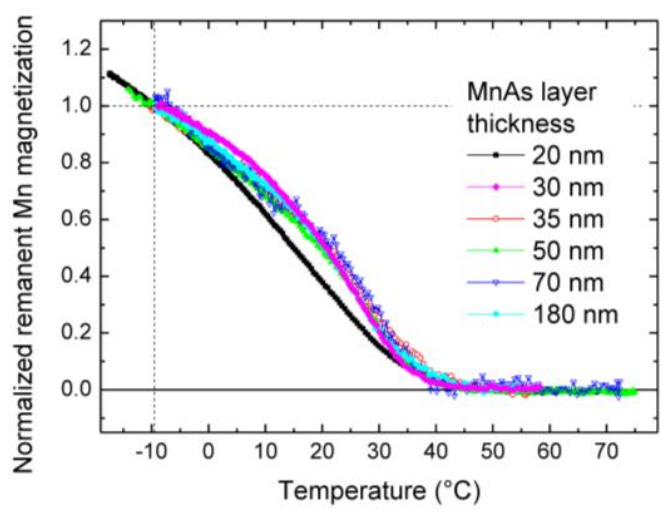

Figure 2. Temperature dependent Mn magnetization curves in a heating cycle, normalized to 1 at $\mathrm{T}=-10{ }^{\circ} \mathrm{C}$. Each curve represents the difference divided by the sum of the Mn-2p resonant scattering intensities collected for opposite orientations between the sample magnetization and the x-ray helicity. The applied field and the MnAs easy magnetization axis are oriented within the $\mathrm{x}$-ray incidence plane.

The curves in figure 2, normalized to 1 at $\mathrm{T}=-10{ }^{\circ} \mathrm{C}$ for better comparison, show an identical behavior as a function of temperature, with only the $t_{\mathrm{MnAs}}=20 \mathrm{~nm}$ sample featuring a slightly different slope. For all samples, the magnetization vanishes at around $40{ }^{\circ} \mathrm{C}$, i.e. at the temperature corresponding to the transition to the $\beta$ phase in bulk MnAs. The magnetization 
curves of figure 2 confirm also that all samples were aligned with their easy magnetization axis within the incidence plane, which is the correct azimuth for observing stripes-related Bragg peaks in our co-planar geometry.

Figure 3 shows the experimental results obtained for the reference $180 \mathrm{~nm}$ thick MnAs sample. The $2 \theta$ scan at fixed $\omega$ [2D-detector acquisition, figure 3(a,b)] shows the presence of Bragg peaks at $\mathrm{q}_{\mathrm{x}} \approx 7 \mu \mathrm{m}^{-1}$ corresponding to a period of $\sim 900 \mathrm{~nm}$, in perfect agreement with the expected $\mathrm{p} \approx 5 \times \mathrm{t}_{\mathrm{MnAs}}$ relationship. The association of the Bragg peaks with the formation of regular $\alpha / \beta$ stripes is further supported by the temperature dependence of their intensity over a heating cycle from 0 to $50{ }^{\circ} \mathrm{C}$, shown in figure $3(\mathrm{c})$.

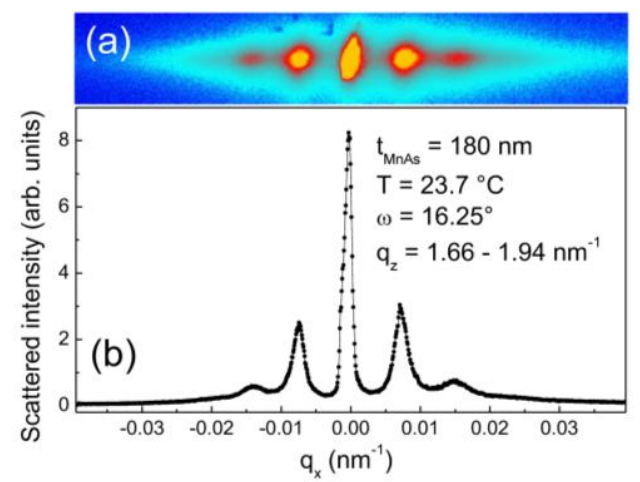

\footnotetext{
Figure 3. Soft $x$-ray scattering results for the $\mathrm{t}_{\mathrm{MnAs}}=180 \mathrm{~nm}$ reference sample. All data were collected at $640 \mathrm{eV}$ photon energy $(\lambda=1.94 \mathrm{~nm})$, corresponding to the $\mathrm{Mn}-2 \mathrm{p}$ resonance. (a) $2 \mathrm{D}$ detector acquisition with $\omega=16.25^{\circ}$. (b) Detector scan curve obtained by vertical integration of (a). (c) Temperature dependence of the $1^{\text {st }}$ order peak intensity.
}

Coming to the thin MnAs layers, figures 4-7 summarize the results for $t_{\mathrm{MnAs}}$ between 20 and 70 nm.

Neither the rocking scans nor the 2D images in figure 4 show any Bragg peak at the expected position for $t_{M n A s}=20 \mathrm{~nm}$. In the $\left(q_{x}, q_{z}\right)$ chart of figure 4(a), no additional intensity corresponding to the expected stripe period is observed at any $\mathrm{q}_{\mathrm{z}}$ value. Figure 4(b) shows identical curves for data collected at $22{ }^{\circ} \mathrm{C}$, where both $\alpha$ and $\beta$ phases coexist (red squares) and at $55{ }^{\circ} \mathrm{C}$, where the MnAs is $\beta$-only and no stripes are expected (black circles). Figure 4(c) compares $2 \theta$ scans recorded at three temperature values in a heating-cooling sequence from 30 ${ }^{\circ} \mathrm{C}\left(\alpha / \beta\right.$ coexistence) to $77^{\circ} \mathrm{C}$ (pure $\beta$ phase) and back to $20^{\circ} \mathrm{C}(\alpha / \beta$ coexistence): no variation can be detected above the noise level. 

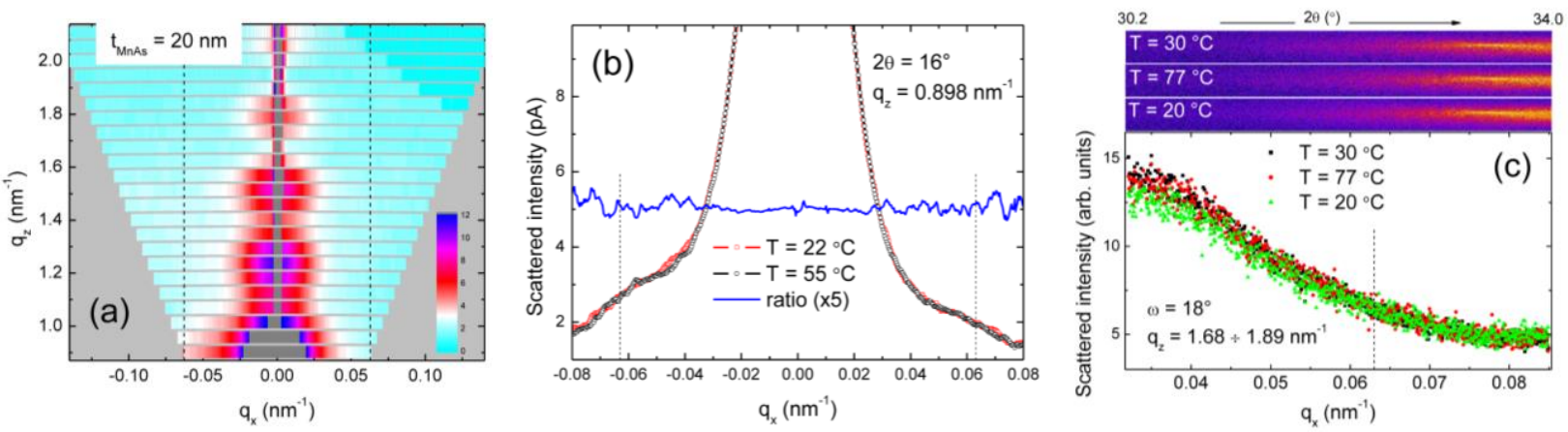

Figure 4. Resonant scattering results for the $t_{\mathrm{MnAs}}=20 \mathrm{~nm}$ sample. Dashed vertical lines locate the expected Bragg peak position for $\mathrm{p}=5 \times \mathrm{t}_{\mathrm{MnAs}}=100 \mathrm{~nm}$. (a) Room-temperature $\left(\mathrm{q}_{\mathrm{x}}, \mathrm{q}_{\mathrm{z}}\right)$ chart obtained by a sequence of rocking scans performed at different $2 \theta$ values. The specular ridge is saturated. (b) Rocking curves at $\mathrm{q}_{\mathrm{z}}=0.898 \mathrm{~nm}^{-1}$ measured at $\mathrm{T}$ $=22{ }^{\circ} \mathrm{C}$ and $\mathrm{T}=55{ }^{\circ} \mathrm{C}$. Their ratio (blue curve, $\left.\times 5\right)$ is also shown. (c) $2 \mathrm{D}$ detector acquisitions $\left(\omega=18^{\circ}\right)$ and corresponding $\mathrm{q}_{\mathrm{x}}$ line profiles measured at three temperatures in the sequence $\mathrm{T}=30^{\circ} \mathrm{C} \rightarrow 77^{\circ} \mathrm{C} \rightarrow 20^{\circ} \mathrm{C}$.

One can draw the same conclusion for the $35 \mathrm{~nm}$ sample, the rocking curves of figure 5(a) and 2D-detector acquisitions of figure 5(b) showing no Bragg peak and no temperature dependence. These measurements demonstrate that no signal can be related to the formation of a temperature dependent ordered microstructure alternating the $\alpha$ and $\beta$ phases in the $20 \mathrm{~nm}$ and $35 \mathrm{~nm}$ thick samples.

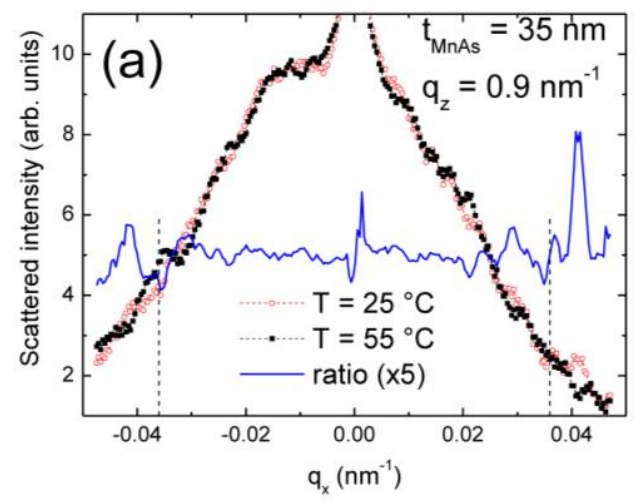

Figure 5. Scattering data for the $\mathrm{t}_{\mathrm{MnAs}}=35 \mathrm{~nm}$ sample. (a) Rocking curves at $\mathrm{T}=25$ and $55^{\circ} \mathrm{C}\left(2 \theta=16^{\circ}\right)$, and their intensity ratio (blue curve, $\times 5$ ). (b) 2D detector acquisitions $\left(\omega=15.25^{\circ}\right)$ and corresponding $\mathrm{q}_{\mathrm{x}}$ line

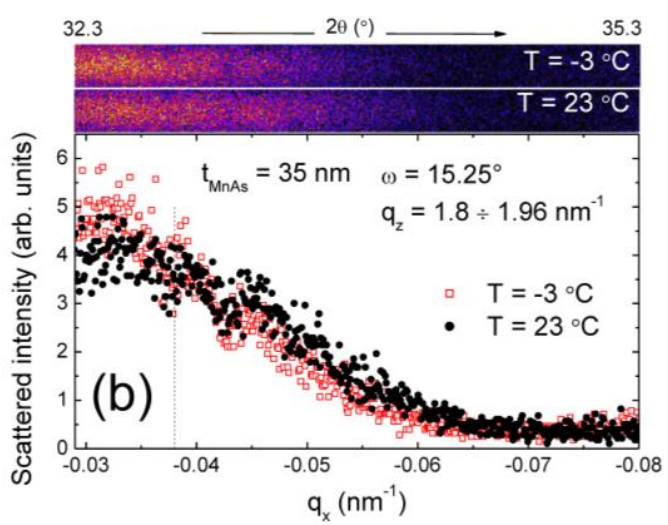
profiles measured at $\mathrm{T}=-3$ and $+23^{\circ} \mathrm{C}$. The vertical lines locate the Bragg peak positions expected for a stripe period $\mathrm{p}=5 \times \mathrm{t}=175 \mathrm{~nm}$. 
The scattering measurements give a different picture for the thicker $50 \mathrm{~nm}$ and $70 \mathrm{~nm}$ samples. Figure 6(a) shows a room-temperature $\left(\mathrm{q}_{\mathrm{x}}, \mathrm{q}_{\mathrm{z}}\right)$ chart for $\mathrm{t}_{\mathrm{MnAs}}=50 \mathrm{~nm}$, featuring diffracted intensity at $\mathrm{q}_{\mathrm{x}}$ values around $\pm 0.024 \mathrm{~nm}^{-1}$, strongly enhanced at $\mathrm{q}_{\mathrm{z}} \approx 0.85 \mathrm{~nm}^{-1}$. Figure $6(\mathrm{~b})$ shows the $2 \mathrm{D}$-detector diagrams measured at $\mathrm{T}=23^{\circ} \mathrm{C}$ and $\mathrm{T}=55^{\circ} \mathrm{C}$, and the corresponding $\mathrm{q}_{\mathrm{x}}$ line profiles. Clear peaks are observed at room temperature, where both $\alpha$ and $\beta$ phases are present, and they disappear when the sample is heated above the temperature for the transition to the $\beta$ phase.
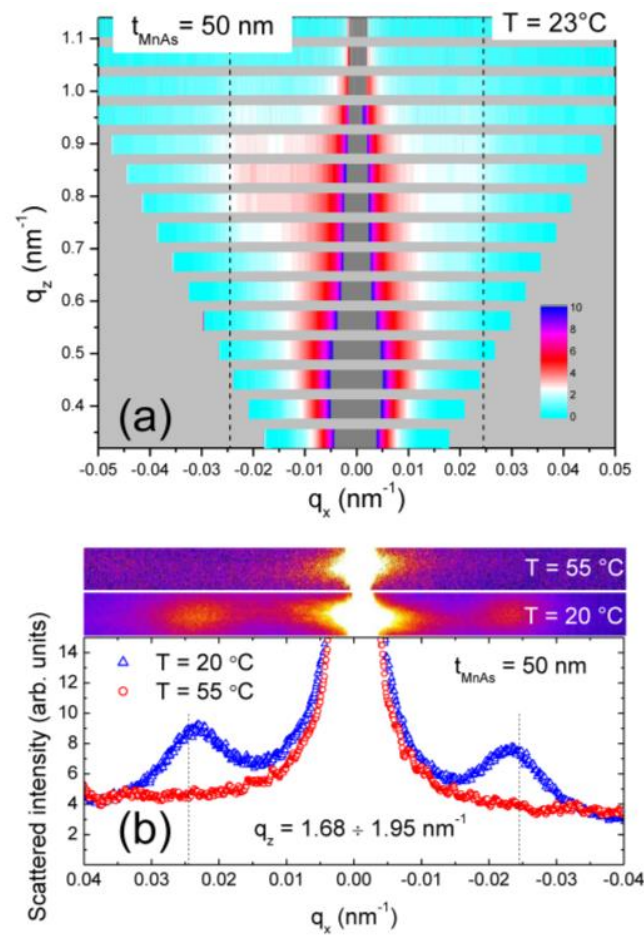

Figure 6. Results of the scattering measurements at 640 $\mathrm{eV}$ photon energy for $\mathrm{t}_{\mathrm{MnAs}}=50 \mathrm{~nm}$. (a) Room-temperature $\left(\mathrm{q}_{\mathrm{x}}, \mathrm{q}_{\mathrm{z}}\right)$ chart (the specular ridge is saturated). (b) $2 \mathrm{D}$ detector measurements $\left(\omega=16.25^{\circ}\right)$ and corresponding $\mathrm{q}_{\mathrm{x}}$ line profiles at $\mathrm{T}=20^{\circ} \mathrm{C}$ and $\mathrm{T}=55$ ${ }^{\circ} \mathrm{C}$. The vertical lines locate the expected peak positions for $\mathrm{p}=250 \mathrm{~nm}$.

Finally, figure 7 shows the results for the $70 \mathrm{~nm}$ sample: strong peaks are measured at room temperature, which disappear by either reducing $\left(\mathrm{T}=-7^{\circ} \mathrm{C}, \alpha\right.$-MnAs only) or increasing $(\mathrm{T}=$ $+57^{\circ} \mathrm{C}, \beta-\mathrm{MnAs}$ only) the temperature.

\section{DISCUSSION}

From the ensemble of the results presented in figures 4-7, we conclude that there is a critical value of the MnAs layer thickness for observing the formation of ordered $\alpha / \beta$ stripes over the temperature region characterized by phase coexistence, and that this value is around $40 \mathrm{~nm}$. In 
the following, we will correlate this finding with data reported previously by other authors and with the results of complementary $\mathrm{x}$-ray diffraction measurements.
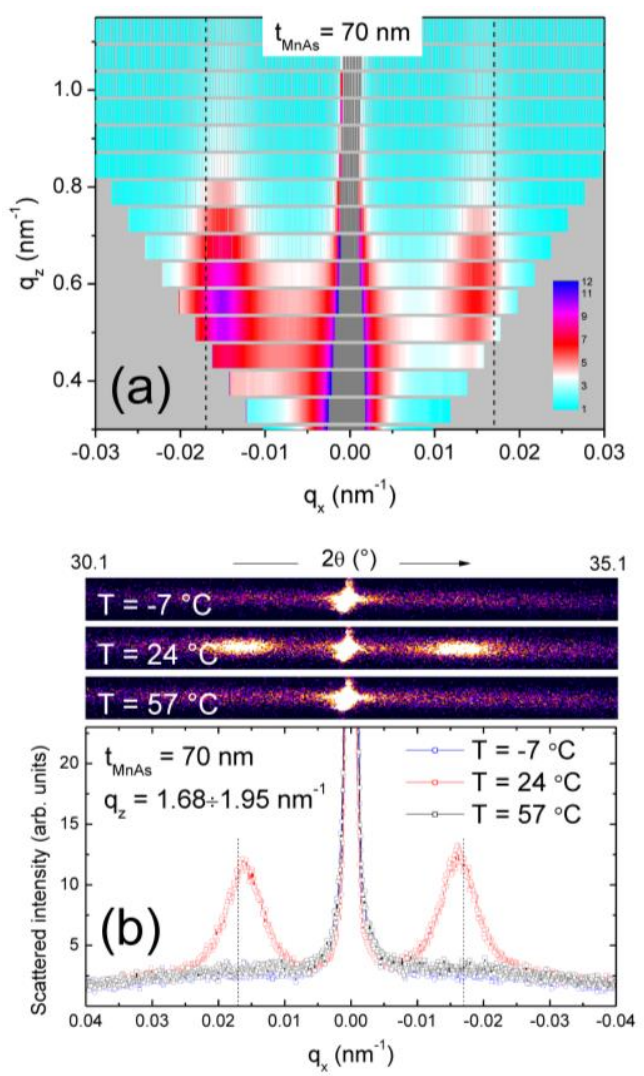

Figure 7. Scattered intensity at $640 \mathrm{eV}$ for the $70 \mathrm{~nm}$ MnAs sample. (a) Room-temperature $\left(\mathrm{q}_{\mathrm{x}}, \mathrm{q}_{\mathrm{z}}\right)$ chart. (b) $2 \mathrm{D}$ detector diagrams $\left(\omega=16.25^{\circ}\right)$ and corresponding $\mathrm{q}_{\mathrm{x}}$ line profiles measured at $\mathrm{T}=-7,24$ and $57^{\circ} \mathrm{C}$. The vertical lines correspond to the peak positions expected for $\mathrm{p}=350 \mathrm{~nm}$.

First of all, one should mention that several studies exist that report magnetic images of thin MnAs layers obtained by either magnetic force microscopy (MFM) or by x-ray photoelectron emission microscopy (X-PEEM). Although none of them addresses the issue of stripe formation versus thickness explicitly, some of the reported results are worth discussing here. In two of the early X-PEEM works on MnAs/GaAs(001), the magnetic images of a $37 \mathrm{~nm}$ [23] and of a $40 \mathrm{~nm}$ [24] layer showed a clear array of nonmagnetic $\beta$ stripes at room temperature. These observations may help us setting an upper limit to the thickness at which ordered stripes are not formed anymore. One must notice, though, that the periods that can be extracted from the X-PEEM images are $\sim 250 \mathrm{~nm}$ for the $37 \mathrm{~nm}$ sample (figure 1 of reference [23]) and $~ 350$ $\mathrm{nm}$ for the $40 \mathrm{~nm}$ one (figure 3 of reference [24]). These values exceed (the former only slightly) what is expected for a $\sim 40 \mathrm{~nm}$ MnAs layer, suggesting that the thicknesses of these samples may had been underestimated.

A much lower value may be inferred from the MFM work of Fraser et al. [26], which mentions the presence of $\alpha / \beta$ stripes with a period of $230 \mathrm{~nm}$ in a $10 \mathrm{~nm}$ thick sample. Unfortunately, no 
image is provided in the article to support this statement. Moreover, such a large period-to-thickness ratio seems unlikely.

A recent X-PEEM study [27] compared room temperature magnetic images for 30 and $50 \mathrm{~nm}$ thick samples (figure 3 of reference [27]). Regular non-magnetic $\beta$ stripes running perpendicular to the easy magnetization direction are evident for the $50 \mathrm{~nm}$ thick MnAs layer, but they are absent in the $30 \mathrm{~nm}$ one. This observation, obtained by a completely different experimental method (X-PEEM), not only agrees with and supports the conclusions of our study, but also proves that these conclusions do not depend on the specific technique (XRMS) that we have employed.

Finally, it should be mentioned also that the investigation of both the magnetic domain structure [25] and of the electrical properties [28] of MnAs films in the low thickness limit suggest a change in behavior across the $20-50 \mathrm{~nm}$ range. These results may be related to microstructural changes and generally agree with our findings.

Based on the results of our work alone, we cannot provide a clear explanation for the absence of ordered stripes below a certain thickness of the MnAs layer. In the following, we will discuss some hypotheses.

The first one concerns a possible relationship with crystalline quality. The initial stages of the MnAs growth on $\mathrm{GaAs}(001)$ were addressed in previous works by following the structural evolution in situ [21, 32]. It was shown in particular that the width of the rocking curves measured at scattering angles that match the MnAs Bragg peaks decreases when the layer thickness increases [33], a result that was associated with the improved grain size, crystal quality and long range order in the film. Therefore, it seems appropriate to question whether the $\sim 40 \mathrm{~nm}$ critical value for observing ordered $\alpha / \beta$ stripes is related to the crystalline quality of the film.

A feature common to all our samples regardless of their thickness is the presence of both $\alpha$ and $\beta$ phases at room temperature. Using $\mathrm{Cu}-\mathrm{K}_{\alpha}$ radiation, we performed $\omega$-scans (i.e., rocking curves) at $2 \theta$ values corresponding to the 006 Bragg peaks (in orthorhombic notation) of the $\alpha$ and $\beta$ phases. Figure $8(a-d)$ shows the rocking scans measured at $2 \theta_{\alpha}$ and $2 \theta_{\beta}$ for four MnAs layers. The $30 \mathrm{~nm}$ sample of figure $8(\mathrm{~d})$ is the same of the X-PEEM experiment of ref. [27]. The value $w$ of the full angular width at half maximum (FWHM), averaged over the $\alpha$ and $\beta$ peaks, is reported for each sample. Figure 8(e) summarizes the $w$ values obtained for a larger set of samples, showing the general trend of a decreasing FWHM as the thickness increases. It is also observed, though, that $w$ values depend strongly on the growth process details: each symbol in 
figure 8(e) identifies samples prepared during a single growth session, showing that, depending on the growth conditions, very small $w$ values can be obtained also for thin layers. The two samples of figure $8(\mathrm{c}, \mathrm{d})$ have similar thicknesses $(35$ and $30 \mathrm{~nm})$ : although they feature rather different rocking curve widths of $0.77^{\circ}$ and $0.36^{\circ}$, stripes are absent for both of them. The samples of figure $8(\mathrm{~b}, \mathrm{c}), 50$ and $35 \mathrm{~nm}$ thick prepared during the same growth session, feature almost identical rocking curve widths $\left(w \approx 0.75^{\circ}\right)$, but behave differently in terms of stripe formation (see figures 5 and 6).

Overall, the ensemble of our data points unequivocally to the absence of a correlation between the crystalline quality of the MnAs layer, at least as measured by $w$, and the presence or absence of ordered $\alpha / \beta$ stripes.



$\omega\left({ }^{\circ}\right)$

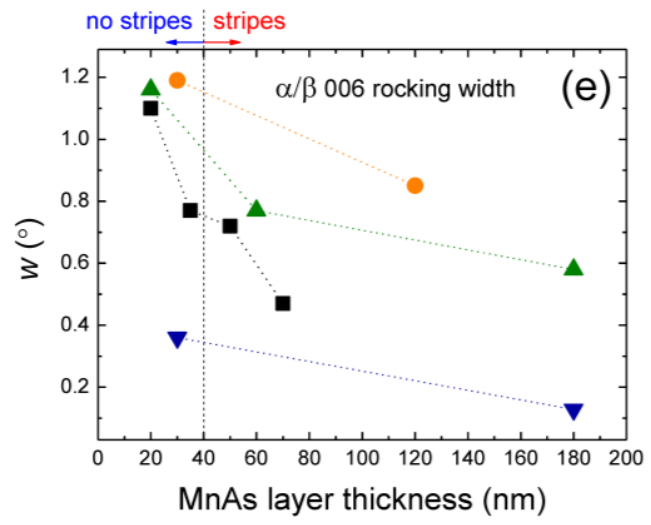

Figure 8. (a-d) Room temperature rocking curves ( $\omega$-scans) measured for four different samples with $2 \theta$ set at the 006 peaks of $\alpha(\square)$ and $\beta(\circ)$ MnAs. The $w$ values reported for each sample correspond to the FWHM averaged for $\alpha$ and $\beta$ peaks. (e) Summary of $w$ values for a wider set of MnAs samples as a function of their thickness. Each symbol identifies a subset of samples prepared during a single growth session. 
Since the step height $\mathrm{h}$ between $\alpha$ and $\beta$ stripes is proportional to the MnAs layer thickness ( $\mathrm{h}$ is $\sim 1 \%$ of $\mathrm{t}_{\mathrm{MnAs}}$ ), one can speculate that reducing the film thickness may make the diffraction peaks too weak to be observed by XRMS. Moreover, also the period $\mathrm{p}$ diminishes with $\mathrm{t}_{\mathrm{MnAs}}$, and in the theoretical work of Kaganer and collaborators [13] it was predicted that the narrowing of the stripes could lead to a further smoothing of the steps between $\alpha$ and $\beta$ domains, accompanied by the convergence towards a common value of the vertical lattice spacing for the two phases. This last prediction is at variance with our observation of clearly distinct $00 l$ diffraction peak positions for $\alpha$ and $\beta$ MnAs down to the thinnest layer that we measured. Moreover, it is worth stressing that the X-PEEM observations mentioned above [27] are not affected by the step height $\mathrm{h}$, since they rely only on the magnetic contrast between ferromagnetic $\alpha$ and paramagnetic $\beta$ phases. Therefore, it seems unlikely that the disappearance of stripes in XRMS data and X-PEEM images when reducing the layer thickness below $40 \mathrm{~nm}$ can originate simply from the reduction of the steps height.

Another possible explanation for the existence of a threshold thickness compatible with experimental observations may come from the loss of the vertical homogeneity in the MnAs layer. Fu et al. [34] report a transmission electron microscopy (TEM) study showing a depth dependent strain field in a $180 \mathrm{~nm}$ thick MnAs lamella, which may lead to an inhomogeneous phase transition. Another TEM work that analyzes the vertical magnetization profile in a 180 nm thick MnAs lamella [35] shows a reduced magnetization at the interfaces with the GaAs(001) substrate and with the capping layer, suggesting a temperature dependent inhomogeneous spatial distribution of the $\alpha$ phase in the layer depth. The impact of such observations would be enhanced for thinner films, where a loss of structural homogeneity in the vertical direction, combined with the smaller lateral size of the domains, may hamper the formation of regular $\alpha / \beta$ stripes. These observations should be tempered by two remarks: i) $\mathrm{Fu}$ et al. conclude that the TEM sample preparation may well be at the origin of the observed change in film properties; ii) inhomogeneous magnetization profiles in MnAs films were reported earlier, but they were attributed to the inhomogeneity of the magnetization vector orientation, rather than to its modulus $[12,36]$, which has only minor or no implication on the structural homogeneity. Finally, let us also remind that our structural and magnetic data show clearly that the $\alpha$-phase persists over the same temperature range regardless of the layer thickness (figure 2), ruling out a premature disappearance of the $\alpha$-phase with temperature in the thinner layers. 


\section{CONCLUSIONS}

From the ensemble of our results we can draw two main conclusions. Firstly, the thickness of the MnAs film certainly matters in defining the presence or absence of regular $\alpha / \beta$ stripes at ambient temperature, with a threshold value that we determine to be around $40 \mathrm{~nm}$. Secondly, the crystalline quality of the MnAs layer, at least as measured by the width of the rocking curves, does not seem to play a fundamental role, if any at all, in the definition of this threshold value.

On the one hand, these results will be very useful in the preparation of future experiments that use the MnAs/GaAs(001) system as the template material for the magnetization control of a ferromagnetic overlayer. In particular, they will be instrumental to envisaging any application that relies on the control of the temperature- or laser-driven surface dipolar fields for attaining magnetization switching. On the other hand, our results do not clarify the mechanisms leading to the existence of a critical thickness. Analyzed together with previously published results, though, they allowed us to discuss and possibly discard some hypotheses.

The most realistic picture for explaining the absence of ordered stripes in very thin MnAs layers seems to involve a loss of vertical structural coherence due to the simultaneous reduction of the thickness and of the lateral domain size. Although this view is not fully supported by experiments yet, it explains the results of all our XRMS measurements and also of previous experiments. Further X-PEEM and sectional TEM images may be helpful to validate this picture. On the theory side, an extension of the model calculations proposed in the seminal work of Kaganer et al. [13] and based on the work of Sridhar et al. [20] would provide a firmer theoretical support for understanding the absence of ordered $\alpha / \beta$ stripes in the low thickness limit of MnAs films on GaAs(001).

\section{ACKNOWLEDGEMENTS}

We acknowledge Sincrotrone Trieste for granting beamtime to our project and the Circular Polarization beamline staff for assistance during the experiment. The research leading to these results has been supported by the project CALIPSOplus under Grant Agreement 730872 from the EU Framework Programme for Research and Innovation HORIZON 2020. We acknowledge the support from the National Natural Science Foundation of China under Grant No. U1632264. 


\section{REFERENCES}

[1] A. V. Kimel, A. Kirilyuk, A. Tsvetkov, R. V. Pisarev, Th. Rasing, Laser-induced ultrafast spin reorientation in the antiferromagnet $\mathrm{TmFeO}_{3}$, Nature 429, 850 (2004). doi: 10.1038/nature02659.

[2] A. Hassdenteufel, B. Hebler, C. Schubert, A. Liebig, M. Teich, M. Helm , M. Aeschlimann, M. Albrecht, R. Bratschitsch, Thermally assisted all-optical helicity dependent magnetic switching in amorphous Fe(100-x)Tb(x) alloy films, Adv. Mater. 25, 3122 (2013). doi: 10.1002/adma.201300176

[3] A. Kirilyuk, A. V. Kimel, Th. Rasing, Laser-induced magnetization dynamics and reversal in ferrimagnetic alloys, Rep. Prog. Phys. 76, 026501 (2013). doi: 10.1088/0034-4885/76/2/026501

[4] S. Mangin, M. Gottwald, C-H. Lambert, D. Steil, V. Uhlír, L. Pang, M. Hehn, S. Alebrand, M. Cinchetti, G. Malinowski, Y. Fainman, M. Aeschlimann, E. E. Fullerton, Engineered materials for all-optical helicity-dependent magnetic switching, Nature Mater. 13, 286 (2014). doi: 10.1038/NMAT3864

[5] J.U. Thiele, S. Maat, E. E. Fullerton, FeRh/FePt exchange spring films for thermally assisted magnetic recording media, Appl. Phys. Lett. 82, 2859 (2003). doi: $10.1063 / 1.1571232$

[6] M. Marangolo, M. Sacchi, Method for changing the direction of magnetization in a ferromagnetic layer. French Patent n. 2947375; US Patent Appl. 13/377, 605 (2012).

[7] M. Tanaka, J. P. Harbison, M. C. Park, Y. S. Park, T. Shin, and G. M. Rothberg, Epitaxial orientation and magnetic properties of MnAs thin films grown on (001) GaAs: Template effects, Appl. Phys. Lett. 65, 1964 (1994). doi: 10.1063/1.112831

[8] F. Schippan, G. Behme, L. Däweritz, K. H. Ploog, B. Dennis, K.-U. Neumann, K. R. A. Ziebeck, Magnetic structure of epitaxially grown MnAs on GaAs(001), J. Appl. Phys. 88, 2766 (2000). doi: 10.1063/1.358304

[9] I. Rungger, S. Sanvito, Ab initio study of the magnetostructural properties of MnAs, Phys. Rev. B 74, 024429 (2006). doi: 10.1103/PhysRevB.74.024429 
[10] J. Łażewski, P. Piekarz, J. Toboła, B. Wiendlocha, P. T. Jochym, M. Sternik, K. Parlinski, Phonon mechanism of the magnetostructural phase transition in MnAs, Phys. Rev. Lett. 104, 147205 (2010). doi: 10.1103/PhysRevLett.104.147205

[11] J. Łażewski, P. Piekarz, K. Parlinski, Mechanism of the phase transitions in MnAs, Phys. Rev. B 83, 054108 (2011). doi: 10.1103/PhysRevB.83.054108

[12] L. Däweritz, Interplay of stress and magnetic properties in epitaxial MnAs films, Rep. Prog. Phys. 69, 2581 (2006). doi:10.1088/0034-4885/69/9/R02.

[13] V. M. Kaganer, B. Jenichen, F. Schippan, W. Braun, L. Däweritz, and K. H. Ploog, Strain-mediated phase coexistence in MnAs heteroepitaxial films on GaAs: An x-ray diffraction study, Phys. Rev. B 66, $045305 \quad$ (2002). doi: 10.1103/PhysRevB.66.045305

[14] F. Vidal, C. Spezzani, R. Breitwieser, M. Marangolo, M. Eddrief, M. Sacchi, V. H. Etgens, Tuning the period of elastic MnAs/GaAs(001) $\alpha \rightarrow \beta$ pattern by Fe deposition, Appl. Phys. Lett. 97, 251914 (2010). doi:10.1063/1.3531759

[15] M. Sacchi, M. Marangolo, C. Spezzani, R. Breitwieser, H. Popescu, R. Dealaunay, B. Rache Salles, M. Eddrief, V. H. Etgens, Thermal switching of the magnetization in an iron film on a magnetically active template MnAs/GaAs(001), Phys. Rev. B 81, 240801(R) (2010). doi: 10.1103/PhysRevB.81.220401

[16] C. Spezzani, F. Vidal, R. Delaunay, M. Eddrief, M. Marangolo, V. Etgens, H. Popescu, M. Sacchi, Thermally induced magnetization switching in Fe/MnAs/GaAs(001): selectable magnetic configurations by temperature and field control, Sci. Rep. 5, 8120 (2015). doi: 10.1038/srep08120

[17] C. Spezzani, E. Ferrari, E. Allaria, F. Vidal, A. Ciavardini, R. Delaunay, F. Capotondi, E. Pedersoli, M. Coreno, C. Svetina, L. Raimondi, M. Zangrando, R. Ivanov, I. Nikolov, A. Demidovich, M. B. Danailov, H. Popescu, M. Eddrief, G. De Ninno, M. Kiskinova, M. Sacchi, Magnetization and microstructure dynamics in Fe/MnAs/GaAs(001): Fe magnetization reversal by a femtosecond laser pulse, Phys. Rev. Lett. 113, 247202 (2014). doi: 10.1103/PhysRevLett.113.247202

[18] F. Vidal, L. Lounis, C. Spezzani, E. Ferrari, R. Delaunay, A. Ciavardini, H. Popescu, M. Eddrief, Y. Zheng, F. Capotondi, E. Pedersoli, C. Svetina, L. Raimondi, M. Zangrando, R. Ivanov, I. Nikolov, A. Demidovich, M. B. Danailov, G. De Ninno, E. Allaria, M. Kiskinova, M. Sacchi, Dynamics of the MnAs $\alpha / \beta$-striped microstructure and of the Fe magnetization reversal in Fe/MnAs/GaAs(001): an optical-laser pump- 
Free-Electron-Laser probe scattering experiment Photonics, 4, 21 (2017). doi:10.3390/photonics4020021

[19] L. Lounis, Y. Zheng, C. Spezzani, E. Ferrari, M. Edrrief, A. Ciavardini, H. Popescu, E. Allaria, C. Laulhé, F. Vidal, M. Sacchi, Dynamics of laser induced magneto-structural phase transitions in MnAs/GaAs(001) epitaxial layers, IEEE Trans. Mag. 53, 8205004 (2017). doi: 10.1109/TMAG.2017.2692209

[20] N. Sridhar, J. M. Rickman, D. J. Srolovitz, Twinning in thin films - II. Equilibrium microstructures, Acta Mater. 44, 4097 (1996). doi: 10.1016/S1359-6454(96)00059-6

[21] D. K. Satapathy, B. Jenichen, V. M. Kaganer, W. Braun, L. Däweritz, K. H. Ploog, In situ investigation of MnAs/GaAs(001) growth and interface structure using synchrotron x-ray diffraction, J. Vac. Sci. Technol. B 22, (2004). doi: $10.1116 / 1.1775200$

[22] J. H. Song, Y. Cui, J. B. Ketterson, Effect of growth temperature on magnetic and electronic properties of epitaxially grown MnAs thin films on GaAs(100) substrates, J. Appl. Phys. 113, 17C307 (2013). doi: 10.1063/1.4801508

[23] L. Däweritz, C. Herrmann, J. Mohanty, T. Hesjedal, K. H. Ploog, E. Bauer, A. Locatelli, S. Cherifi, R. Belkhou, A. Pavlovska, S. Heun, Tailoring of the structural and magnetic properties of MnAs films grown on GaAs - Strain and annealing effects, J. Vacuum Sci. Technol. B 23, 1759 (2005). doi: 10.1116/1.1978902.

[24] R. Zdyb, A. Locatelli, S. Heun, S. Cherifi, R. Belkhou, E. Bauer, Nanomagnetism studies with spin-polarized low-energy electron microscopy and $x$-ray magnetic circular dichroism photoemission electron microscopy, Surf. Interface Anal. 37, 239 (2005). doi: 10.1002/sia.1973

[25] K.-S. Ryu, J. B. Kim, Y. P. Lee, H. Akinaga, T. Manago, R. Viswan, S.-C. Shin, Thickness-dependent magnetic domain change in epitaxial MnAs films on GaAs(001), Appl. Phys. Lett. 89, 232506 (2006). doi: 10.1063/1.2402235

[26] E. D. Fraser, C. H. Kim, S. Hegde, H. Zeng, H. Luo, P. K. Wei, Magnetization reversal in epitaxial MnAs thin films, J. Appl. Phys. 104, 033921 (2008). doi: $10.1063 / 1.2966672$

[27] L. B. Steren, M. Tortarolo, F. Fernandez Baldis, M. Sirena, M. Sacchi, V. H. Etgens, M. Eddrief, B. Santos, T. O. Mentes, A. Locatelli, Combined effects of vertical and lateral confinement on the magnetic properties of MnAs micro and nano-ribbons, J. Appl. Phys. 120, 093905 (2016). doi: 10.1063/1.4961501 
[28] F. Fernandez Baldis, M. Sirena, L. B. Steren, V. H. Etgens, M. Eddrief, C. Ulysse, G. Faini, Anisotropic magnetic-field-induced phase transition in MnAs nanoribbons, Appl. Phys. Lett. 107, 012407 (2015). doi: 10.1063/1.4926567.

[29] M. Sacchi, M. Marangolo, C. Spezzani, L. Coelho, R. Breitwieser, J. Milano, V. H. Etgens, Uniaxial anisotropy and temperature driven magnetization reversal of $\mathrm{Fe}$ deposited on a MnAs/GaAs(001) magnetic template, Phys. Rev. B 77, 165317 (2008). doi: 10.1103/PhysRevB.77.165317

[30] F. Vidal, Y. Zheng, L. Lounis, L. Coelho, C. Laulhé, C. Spezzani, A. Ciavardini, H. Popescu, E. Ferrari, E. Allaria, J. Ma, H. Wang, J. Zhao, M. Chollet, M. Seaberg, R. Alonso-Mori, J. Glownia, M. Eddrief, M. Sacchi Ultrafast structural dynamics along the $\beta$ - $\gamma$ phase transition path in MnAs Phys. Rev. Lett. 122, 145702 (2019). doi: 10.1103/PhysRevLett.122.145702

[31] M.Sacchi, C.Spezzani, P.Torelli, A.Avila, R.Delaunay, C.F.Hague Ultra-high vacuum soft X-ray reflectometer Rev. Sci. Instrum. 74, 2791 (2003). doi: $10.1063 / 1.1568552$

[32] M. Hirayama, G. R. Bell, S. Tsukamoto, Initial stages of MnAs heteroepitaxy and nanoisland growth on GaAs(110) and (001)surfaces, J. Vac. Sci. Technol. B 29 (2011). doi: 10.1116/1.3610963

[33] B. Jenichen, D. K. Satapathy, W. Braun, V. M. Kaganer, L. Däweritz, K. H. Ploog, Structural properties of MnAs epitaxial films on GaAs: an in situ x-ray study, J. Phys.

D: Appl. Phys. 38, A169 (2005). doi:10.1088/0022-3727/38/10A/032

[34] X. Fu, W. Ye, N. Zhou, Dimension effect on the in-depth phase transition of MnAs/GaAs(001): From a thin film to a nanorod, AIP Adv. 8, 075110 (2018). doi: $10.1063 / 1.5034207$

[35] C. Gatel, X. Fu, V. Serin, M. Eddrief, V. Etgens, B. Warot-Fonrose, In depth spatially inhomogeneous phase transition in epitaxial MnAs film on GaAs(001), Nano Lett. 17, 2460 (2017). doi: 10.1021/acs.nanolett.7b00144

[36] R. Engel-Herbert, J. Mohanty, A. Ney, T. Hesjedal, L. Däweritz, K. H. Ploog Understanding the submicron domain structure of MnAs thin films on GaAs(001): Magnetic force microscopy measurements and simulations, Appl. Phys. Lett. 84, 1132 (2004). doi: 10.1063/1.1645328 